\begin{abstract}
Objective: The purpose of this study was to explore mothers' experiences of negative emotions during breastfeeding and to understand the meaning and consequences that such experiences had on mothers' sense of self and the relationships they formed with their children. Design: A qualitative design was applied to this study as it was judged as the most appropriate approach to this novel field of enquiry. Setting: The study was conducted in United Kingdom using a sample of mothers drawn from five different countries from Europe, America and Australia. Participants: The sample consisted of 11 mothers who reported experiencing or having experienced negative emotions associated with breastfeeding in the past five years. Measurements: Semi-structured interviews were conducted with the mothers and interviews were transcribed to enable the process of data analysis. Interpretative Phenomenological Analysis (IPA; Smith et al., 2009) was chosen as a method of data analysis, enabling in depth understanding and interpretation of the meaning of mothers' experiences. IPA was chosen due to its idiographic commitment and particular interest in sense-making, phenomenology and hermeneutics. Findings: Through the analysis process, three themes were generated reflecting the multifaceted nature of breastfeeding experiences (i) 'Breastfeeding: An unexpected trigger of intense embodied emotional sensations incongruent with view of self', (ii) 'Fulfilling maternal expectations and maintaining closeness with the child', (iii) 'Making sense of embodied emotional sensations essential to acceptance and coping'. Conclusions: Breastfeeding has the potential to trigger a range of conflicting cognitions and emotions in mothers that may impact on how mothers view themselves and relate to their children. Implications for Practice: Increasing awareness about emotional breastfeeding experiences and recognising the multifaceted, individual nature of difficulties around breastfeeding enables professionals to offer mothers person-
\end{abstract}


centred care and avoid making clinical decisions and recommendations based on inaccurate knowledge.

Mothers’ Experiences of Negative Emotions while Breastfeeding: An Interpretative Phenomenological Analysis

Breastfeeding is associated with a number of short and long-term benefits for both the mother and baby (Department of Health [DoH], 2007; National Institute for Health and Clinical Excellence [NICE], 2008; World Health Organization [WHO], 2009; Cesar et al., 2016; Rollins et al., 2016). In the comparatively wealthy, industrialised cultural context of this research, breastfeeding is primarily promoted for the health of the infant. However, breastfeeding has also been argued to fulfil a number of psychological functions for breastfeeding mothers (Marshall et al., 2007). These may include soothing and connecting with the infant (Wiessinger et al., 2010), meeting expectations of motherhood (Hauck and Irurita, 2003) and being a source of women’s maternal identity (Larsen et al., 2008; Huang et al., 2012).

Feeding an infant from the breast is the biological norm for humans (Wiessinger, 1996) and an evolutionary adaptive relationship that ensures survival of the infant (Wiessinger et al., 2010). While the wide ranging benefits of breastfeeding are well established, breastfeeding is not regarded as the societal norm for infant feeding in many parts of the world (Rollins et al., 2016). In western culture, for example, breastfeeding frequently portrayed, through media as well as academic research, as an infant feeding practice that is 'optimal', 'perfect’ or 'ideal' (Wiessinger, 1996; Cattaneo, 2008; Smith et al., 2009). As a corollary of this, formula feeding has become the norm for infant feeding in western culture, which will affect mothers' decisions about how to feed their infants (Wiessinger, 1996). Indeed, the meaning that a mother ascribes to infant rearing practices, 
such as feeding, will be reinforced by her own mental representation of being mothered and influenced by a range of factors in her past and present circumstances (Raphael-Leff, 2001) and the cultural context around her (Thomson et al., 2015).

A commonly cited reason for mothers choosing to breastfeed is that it enhances bonding between them and their infant (Wiessinger et al., 2010). This has been explored in research looking at the role that oxytocin plays in breastfeeding, touch and human relationships (Uvnäs-Moberg, 1998, 2009; Carter, 2003). Strathearn et al. (2009) found that mothers who had an increased response to oxytocin showed activation of brain regions associated with pleasure and rewards when interacting with their infants. Another study linked lower oxytocin levels during breastfeeding to elevated anxiety and depression scores which, in turn, may impact on mothers' vulnerability to postnatal depression and problems related to breastfeeding (Stuebe et al., 2013).

Although the above evidence points to breastfeeding offering the basis for normal, adaptive physiological and psychological development to take place, the breastfeeding relationship is not always experienced this way for a number of dyads. Breastfeeding is frequently portrayed as a natural skill (Phillips, 2011) rather than a competency to be learnt by the mother (Avery et al., 2009), and this perspective omits the difficulties that establishing a breastfeeding relationship may involve (Larsen et al., 2008).

In fact, a number of mothers report emotional challenges during feeding in the absence of, or unrelated to, physical breastfeeding problems. This might involve strong embodied emotional sensations of irritation, aggression or disgust, not uncommonly displaced onto the nursing infant or child, an experience that is referred to as breastfeeding/nursing aversion or agitation in breastfeeding communities (Flower, 2003). Such experiences have been discussed on blogs and breastfeeding forums on parenting 
websites but no academic, peer-reviewed published resources exist to further elaborate on this experience.

Another example of such embodied emotional sensations during breastfeeding is the experience during which mothers report a marked drop in mood occurring a short period of time before milk release during breastfeeding, following which they experience wide ranging mind states including hopelessness, hollowness, anxiety, shame, dread and even suicidal ideation (Heise, 2011). This set of experiences has been informally referred to as Dysphoric Milk Ejection Reflex (D-MER) in a number of Internet-based breastfeeding communities (Heise, 2011; Heise and Wiessinger, 2011) and D-MER has been documented in two case studies independent of each other (Cox, 2010; Heise and Wiessinger, 2011). Heise and Wiessinger (2011) propose D-MER to be distinct from a general dislike of breastfeeding or the experience of postnatal depression (PND). Indeed, PND is associated with persistent experiences of low mood over a substantial period of time (NICE, 2014) and not specifically in relation to feeding. However, PND and negative feelings during feeding may coexist (Heise, 2011). While research suggests that survivors of sexual childhood abuse may find breastfeeding a challenging experience (e.g., Coles, 2009; Klaus, 2010) existing case studies on embodied emotional sensations during breastfeeding, as defined in this study, has not found links between such experiences and repressed sexual trauma (Cox, 2010).

\section{A Note on the Use of Terminology}

For the purpose of this research, the term embodied emotional sensations will be used to denote the marked or sudden emergence of the above described distressing emotional and visceral experiences (e.g., sadness, anger or anxiety) specifically associated with milk-release or the breastfeeding act. Furthermore, the term western culture denotes the comparatively wealthy, industrialised cultural context of this research. 


\section{Rationale and Objectives}

Investigating the experience of embodied emotional sensations during breastfeeding may have implications for the following reasons. In a western cultural context maternal expectations of breastfeeding and their expectations of motherhood are often reciprocally related (Hauck and Irurita, 2003; Marshall, 2011), like “two sides of the same coin” (Larsen et al., 2008, p. 655). When mothers take personal responsibility for breastfeeding to be successful, an inability to meet such expectations can have deleterious consequences for their sense of self (Murphy, 2000; Larsen and Kronborg, 2012; Redshaw and Henderson, 2012).

Secondly, research suggests that mothers, whose expectations around the perinatal period have not been met, are more likely to struggle with postpartum adjustment (Lewis and Nicolson, 1998; Pancer et al., 2000). For example, a recent study of nearly 14,000 mothers found that breastfeeding significantly reduced the risk of PND for mothers who had planned to breastfeed and successfully managed to do so, whereas mothers who had planned to breastfeed, but had to wean prematurely, were at increased risk of PND (Borra et al., 2014). Thus, supporting mothers, who want to breastfeed, to be successful is a way to support mothers post birth, at a time when many women feel vulnerable in their mothering role (Kleiman and Wenzel, 2009).

The main objective of this research was to gain a better understanding of mothers’ experiences of negative emotions during breastfeeding and used the following research questions as a basis for inquiry: 'How do women experience negative emotions during breastfeeding?', 'How, if at all, do such experiences influence maternal well-being and sense of self?' and 'What consequences, if any, do mothers perceive that their experiences have had for the bond between them and their children?'.

\section{Methodology}




\section{Design}

The aim of this study was to obtain a preliminary understanding of how mothers experience distressing emotions during breastfeeding, thus the study design included a qualitative approach (Sofaer, 1999; Smith and Osborn, 2008; Harper, 2012; Thompson and Harper, 2012). More specifically, semi-structured interviews were combined with Interpretative Phenomenological Analysis (IPA; Smith et al., 2009) which was chosen due to its idiographic focus (Shaw, 2010) and particular concern with how individuals experience and make sense of specific experiences at a given time point (Pietkiewicz and Smith, 2012; Starks and Trinidad, 2014).

\section{Recruitment and Participants}

The criteria for inclusion in the study were mothers who were currently breastfeeding or had done so in the past five years; who had experienced negative emotional sensations during breastfeeding; who were able to undertake an interview in English, and who were located within travelling distance of the researcher or had access to telephone or Skype. Mothers were not excluded if they had experienced mental health difficulties, such as PND. Previous case studies (Cox, 2010; Heise andWiessinger, 2011) highlight that although negative emotions during breastfeeding may coexist with PND the former difficulties are experienced markedly differently by mothers due to their reported visceral and unexpected nature.

The study was reviewed and approved by the researchers' host institution ethics process. Mothers were recruited worldwide across five online parenting forums. They were invited to participate through an advertisement raising awareness about the study which was posted in the parenting communities. The final sample consisted of 11 mothers, a number which was sufficient to explore convergences and divergences across accounts while 
retaining the idiographic focus of the research (Smith et al., 2009). Please see Table 1 for a summary of participant demographics.

Insert Table 1

\section{Data Collection}

Participating mothers were interviewed through various means; four face-to-face, one via phone, and six via Skype. Research has documented benefits of using Skype in contact with participants, allowing participants to remain in their preferred location, overcoming geographical distances and simulating a face-to-face contact (Hanna, 2012; Svensson et al., 2014). Thus, such methods were deemed equivalent in terms of the purpose of the interviews which was gaining an understanding of the experiences and meanings that negative emotions during breastfeeding had for mothers. Written consent was obtained for all participants. All interviews were conducted at a mutually convenient time and place. For ten mothers the preferred setting for interview was in their homes, while one mother preferred to meet at a local café.

Data were collected through semi-structured interviews with six broad questions, prompts and probes to encourage conversation around the research aims (see Appendix A). Interviews, which lasted between 35 and 58 minutes (average 46 minutes), were audio recorded. Throughout data collection the inter-relational nature of interviewing was acknowledged and its influence on the interview process considered (Ellet, 2011). Although it is not possible, nor congruent with IPA, to fully detach from held theoretical and personal beliefs (Dahlberg, 2006; Snelgrove, 2014; Probst, 2015), such beliefs can be bracketed or bridled to avoid misleading the research process based solely on such beliefs (Dahlberg, 
2006). For the current study, such bracketing/bridling included taking a reflexive stance throughout the process of data collection and write up. The keeping of a reflexive journal allowed for thoughts and feelings to be recorded and reflected on, considering how to best make use of them to benefit the research process (Creswell, 2012).

\section{Data Analysis}

The transcripts were initially analysed independently to retain the idiographic aspects of participants' accounts (Snelgrove, 2014). Each transcript was scanned for information relevant to the research questions. Notations were made, capturing the essence of participants' views, beliefs or experiences, both in terms of descriptions and meanings. Next, notations were grouped into themes for each participant. When all transcripts had been individually coded, second iteration themes were developed by considering the similarities and differences between first iteration themes across transcripts. Finally, a similar process took place for the third iteration superordinate themes. For the sake of transparency, an audit trail of the analysis process (Snelgrove, 2014; Probst, 2015) is offered in the appendices (Appendix B) outlining how first, second and third order interpretations were developed, and also which participants contributed to each of the themes.

\section{Findings and Discussion}

The process of data analysis generated three themes which offer a preliminary understanding of how mothers in this study navigated embodied emotional sensations during breastfeeding in the context of what breastfeeding meant to them. Themes will be outlined, 
supported by original quotes ${ }^{1}$ from participants ${ }^{2}$ and discussed in relation to existing literature.

\section{Theme 1: Breastfeeding - An Unexpected Trigger of Intense Emotional Embodied Experiences Incongruent with View of Self}

Breastfeeding was an unexpected trigger of intense embodied emotional sensations, thoughts and behaviours that mothers regarded as contrasting with their view of themselves. Milk release or the physical act of breastfeeding evoked a strong, negative bodily response that mothers found difficult to comprehend and communicate: "If I try to think about it with my brain, it just doesn’t work, I can’t really put words to it” (Ciara). Their choice of words such as "visceral” (Kate) and "physical reflex" (Donna) together with vivid descriptions such as “skin crawling” (Leanne), “throat tightening” (Izabella) or “gut wrenching” (Donna) further embodied their experience: “Every time I’d get let-down [milk release], I would start crying (...) so it was like I got let-down from both” (Kate).

Feelings were intense and convincing, however, they were incongruent with the context which mothers currently found themselves in and this gave rise to them secondguessing their emotional experiences:

It just felt like something terrible just had happened and I needed to cry about it but nothing terrible did happen, so that was kind of hard to place for me as well, because I had, I was just nursing my baby, not experiencing some, eh, tragedy or something, but it felt like that. (Ellen)

\footnotetext{
${ }^{1}$ For clarification, symbols used within participant quotes have the following meaning: Three ellipsis points (...) indicate that some text has been omitted; square brackets [ ] contain the researcher's words aimed to clarify/contextualise the content; and round parentheses () contain non-verbal communication.

${ }^{2}$ In line with the British Psychological Society's code of research ethics (BPS, 2010) all real names have been replaced by pseudonyms.
} 
Embodied emotional sensations during breastfeeding characterised by anger or irritation were particularly conflicting for mothers who highly valued and prioritised closeness and attachment between mother and child. During breastfeeding they described not wanting to be near their nursing child: "It's such a strong feeling of 'get away from me'” (Cecile) and “it just kind of feels, I don’t know, like, like I never want to be touched again” (Leanne).

When mothers experienced emotions that felt alien to their beliefs and ideals, they separated themselves from them. Negative embodied emotional sensations during breastfeeding were described as "irrational” (Chloe) and "not really feelings of my heart" (Cecile) while at the same time their intensity and power were compelling: "It [the anger] made me do things I don't want to do” (Ellen). For some mothers the discovery of such unexpected emotional reactions gave rise to negative evaluations of themselves. This included a sense of "failure" (Renee) and viewing their emotional reactions as flaws inherent in themselves: “I can’t help but sometimes feel like there’s something incredibly wrong with me that my body doesn't work the way it's supposed to, that I should be able to breastfeed my son and enjoy it” (Melissa).

When difficulties around breastfeeding compromised mothers' sense of self they felt overcome by a loss of control and anger, further perpetuating a negative sense of self:

It was just that moment and when the nursing session was done I was so angry at myself [that] I couldn’t control it (...) like I couldn't believe that I'm a grown woman and I can’t control those feelings. (Danielle)

Such experiences provided mothers with an interpretive lens through which they evaluated themselves as being "less immune” (Izabella) to everyday challenges, “doubted self as a mother” (Donna) and even mimicked previous difficult experiences: 
When I was younger I suffered really big depression so it kind of felt the same and the thoughts that came in to my head were oh my god I'm going to have another depression, it's going to last forever, I'm never going to get better. How can I be a mum when I'm so sick again? And well, it all seemed so dark um, with no light at the end of the tunnel. (Ellen)

In summary, existing research on breastfeeding has highlighted how physical breastfeeding problems can give rise to guilt, a sense of inadequacy and failure (Kelleher, 2006; Hegney et al., 2008). Additionally, the current findings uniquely brought awareness to breastfeeding as a potential trigger for psychological responses in absence of physical breastfeeding problems. Strong embodied emotional sensations created secondary psychological responses in these mothers, which led to them separating themselves from their emotions or negatively evaluating themselves as inherently flawed. Embodied emotional sensations during breastfeeding provided a lens through which mothers viewed their day-today struggles as overwhelming and for some, mimicked existing vulnerabilities like depression.

\section{Theme 2: Fulfilling Maternal Expectations and Maintaining Closeness with the Child}

All of the mothers held breastfeeding in high esteem, for pragmatic and health reasons but also because of the importance that breastfeeding played in their mothering identity. Breastfeeding was described as a “motherly duty” (Cecile), “mum’s job” (Chloe) and “the best thing I should be doing” (Renee). For Ellen, breastfeeding also provided her with evidence of her worth as a mother:

[Breastfeeding] is the only proof, touchable proof, that I'm a good mother, so (...) if I stop nursing then I don't have any proof for myself that I'm a good mother, but 
obviously it’s ridiculous, I know, but I think that feeling makes me wanting [sic] to not stop nursing. (Ellen)

Consequently, several mothers persevered to uphold their view of themselves as committed to their original choices and beliefs: "He [husband] was worried about me and he wanted me to give up (pause) and then I was being stubborn, saying no because this is the best thing for her [baby]” (Renee).

Mothers reported an ambivalent relationship to breastfeeding and becoming emotional during breastfeeding also made them receptive to the positive feelings associated with the act:

Because right together with it [the negative feeling] comes another feeling, like very close attachment to the baby, like I care a lot about my baby (pause). I feel like, the (pause) love (giggles) or the butterflies as well. (Izabella)

Indeed, the experience was viewed as “too valuable to take away” (Melissa) and mothers’ wish to feed their children outweighed the distress associated with it: “I don’t want to take that away from him and a few more minutes of discomfort for me, is kind of a small price to pay” (Leanne).

Some of the mothers continued to breastfeed despite feeling negative emotions in order to compensate for difficult beginnings or life transitions. Chloe, for example, whose daughter was born prematurely, was determined to persevere with breastfeeding because she was "trying to be as perfect as [she] could be with her [daughter]". Mothers also reported using breastfeeding to compensate for the relationship strains and ruptures which had initially emerged from the breastfeeding problems themselves: 
I feel like if anything after, after I, you know, treated him more roughly than I wanted to then of course, then I wanted to nurse him and tried to make it all better, and that was difficult too. (Cecile)

Some mothers persevered with breastfeeding in the hope that it would bring back the breastfeeding relationship that they once had: “I think that's another reason why (...) I persevered is because I looked forward to having maybe what we did have, as a breastfeeding relationship again” (Melissa). However, mothers reported disappointment with the unexpected turn of events, where the act of breastfeeding, which was meant to offer closeness and increase the bond between mother and child, frequently had the opposite effect:

He will come and sit on my lap, and he will need affection and love and cuddles, and he'll ask for milk. Now, I really want to give affection, love and cuddles, but I may not want to give the milk, so I end up inadvertently rejecting him. (Ciara)

Rectifying a strained breastfeeding relationship was particularly important to mothers who perceived that their feelings had negative consequences extending beyond the feeding relationship, such as getting in the way of desired parenting:

I’m snapping more at my oldest child (...) it doesn’t improve my relationship, it doesn't make me a nicer mum (...) because when I'm nursing and I'm feeling this anger and then later on snapping to my oldest one I feel really guilty because it's not his fault, but I am snapping at him, for feeding his, for nursing his younger brother. (Ellen)

Melissa’s retrospective understanding was: “Honestly, looking back now I probably would have weaned him entirely, because for a good while our relationship was extremely strained (...) because he would get extremely upset” (Melissa). 
In summary, mothers spoke of breastfeeding fulfilling both physiological and nutritional needs of their infants/children while also highlighting and the relational value that breastfeeding had for them. Research has demonstrated how the physical act of breastfeeding takes on a deep seated emotional meaning for many mothers, making the biological and psychological aspects of feeding inseparable (Palmér et al., 2010). Ryan et al. (2011) also interpreted mothers’ views of breastfeeding as an “interembodied experience” (p. 737) leading to mutual fulfilment between mother and infant and a means to communication between them (Palmér et al., 2010). However, extending from this research, current findings also showed how, in addition to feelings of fulfilment, breastfeeding can lead to ambivalent emotional responses that emerge in close succession of each other.

Mothers spoke about breastfeeding as a fundamental aspect of their maternal identity and worth, where failing at breastfeeding was equivalent to failing at motherhood. This echoes existing research suggesting that mothers' expectations of breastfeeding and mothering are intimately linked (Hauck and Irurita, 2003; Marshall, 2011) and that a negative sense of self may follow if they do not manage to successfully breastfeed (Murphy, 2000; Larsen and Kronborg, 2012; Redshaw and Henderson, 2012). However, current findings portray how, in addition to fulfilling maternal expectations, breastfeeding served the function of compensating for imperfections in the relationship between mother and child, such as premature birth or adjusting to new siblings in the family. In addition, current findings highlighted how negative embodied emotional sensations during breastfeeding had the potential to put strain on the mother-child relationship and sending out conflicting messages of closeness, which was in stark contrast to the initial reasons why mothers chose to breastfeed.

Theme 3: Making Sense of Embodied Emotional Sensations Essential to Acceptance and Coping 
Seeking support and information about their experiences from a number of sources enabled mothers to better understand and manage their experiences. However, they all reported disappointment with the scarcity of information that was available to them.

A number of mothers experienced that professionals used existing knowledge and frameworks to interpret mothers' unusual experiences, confusing embodied emotional sensations during breastfeeding with postnatal depression:

They [professionals] did offer me things like antidepressants and things, which I didn't think I needed. I didn’t think that was what (pause) was causing it because I wasn't like that all the time. It was just when I was feeding her (...) I didn’t think it was postnatal depression. (Renee)

In addition, the power imbalances and safeguarding procedures inherent in healthcare services, as well as lack of trust in professionals, impeded disclosure:

I think it's really hard to find a care provider that you can go to and say you, you know, 'I'm angry and I had this urge to throw my kid across the room' without them saying, 'well, we need to call CPS [Child Protection Services]' (...) I never actually would have or had the actual intension of hurting my child. (Melissa)

A number of mothers expressed their perception that breastfeeding promotion and antenatal care typically offer an idealistic picture of breastfeeding and that misleading information can have deleterious consequences for mothers' sense of self and their breastfeeding experience:

“All you ever hear [is] how breastfeeding is brilliant, perfect, and you're really happy when you breastfeed, and (...) some people are made to feel like they're weird because you don’t have an oxytocin high.” (Donna) 
Consequently, a number of mothers reported profound relief when they came across support groups or other mothers with similar experiences, as it alleviated their sense of defectiveness and failure at motherhood. Ellen said "It was like coming home (...) More souls who understand my feeling. I'm not crazy, I'm not a bad mother”. Meeting others with similar experiences, not only served a normalising function, but offered a "safe” (Leanne) space for talking about those experiences.

Mothers who did not access support groups or who did not meet other mothers with similar experiences sought information in breastfeeding literature or online. For Donna, identifying and understanding the experience as one which affected other mothers changed how she perceived it and reduced the power it had over her:

When I kind of had researched it a bit, I knew the reason for it and I knew it wasn't me being abnormal or weird, you know, and I knew of other people that it happened to so it felt more (pause) I just kind of accepted it (...) it stopped bothering me that, I think when I could have a scientific, em, name and an explanation for it. (Donna)

Regardless of source of information, all mothers made sense of their experiences as resulting from hormonal activity associated with milk release or the physical act of breastfeeding. Having professionals to support such claims further added credibility to mothers’ own explanations:

It seemed to be such a strong hormonal response in me, because I had, um, pre-natal depression in my second trimester as well and they [health professionals] said it's sort of classic signs that the hormones are sending me a bit crazy. (Kate)

Some mothers found relief in putting an evolutionary explanation to their experiences: 
It feels like my body is rejecting the, the, the milk theft, (both laugh quietly) from the other child so the milk is actually being directed away from my little baby who needs it, and that's being expressed by my body like a physical revulsion. (Ciara)

Understanding their experiences as mammalian instincts or hormonal activity served a non-blaming and de-shaming function for mothers, allowing them permission to externalise the cause of their experiences: “It was just a biological thing that I couldn’t really control” (Leanne). However, when mothers began to observe that the intensity of their experiences fluctuated depending on contextual factors such as being "stressed" (Izabella) or "tired" (Ciara) they were able to regain a sense of ownership of their well-being: "If I took my vitamins, I ate well and I was able to sleep at least a good amount, I felt less irritable and, like, I was able to control it a little more” (Danielle).

In summary, mothers sought formal and informal sources of support to alleviate their distress caused by negative embodied emotional sensations and to gain a better understanding of their experiences, enabling them to cope. They felt disappointed with professionals' lack of knowledge and instead turned to support groups and literature. Coming across explanations of breastfeeding struggles as partially biological or hormonal served a normalising and de-shaming function for mothers. While Ussher (2003) has argued that when women label their experiences as biological 'symptoms' separate from themselves, it may lead them to a sense of powerlessness. However, current findings also highlighted how understanding negative breastfeeding experiences as a biological phenomenon served as a coping mechanism to avoid guilt and self-blame. Indeed, research suggests how increased awareness and normalisation of a phenomenon can have an ameliorating effect on how it is experienced, because it opens up an individual to proactively engaging in coping strategies that reduce distress associated with that experience (Ussher and Perz, 2013). 
While breastfeeding is not the cultural norm in western society, the majority of mothers in this study did, in fact, approach breastfeeding as the unquestionable way to feed their infants. Thus, when faced with extraordinary negative emotional experiences, their default position was to creatively seek out a number of ways to cope before considering weaning and thus, persevered with breastfeeding for many months. It may be possible that if breastfeeding was culturally accepted as the norm for infant feeding, mothers may draw on a range of sources, other than weaning, to understand, manage and accept their experiences.

\section{Clinical Recommendations}

Mothers in the current study expressed disappointment with professionals’ lack of knowledge about embodied emotional sensations during breastfeeding which compromised mothers' belief that they would be adequately understood and supported in the healthcare system.

Consequently, training for professionals who come into contact with mothers in the perinatal period could benefit from approaching breastfeeding as the biological norm that it is (Wiessinger, 1996). This could include approaching the range of breastfeeding problems that mothers may experience (physical and psychological) as a natural part of the breastfeeding relationship. Health professionals are often perceived as sources of knowledge and reliable information (Hauck and Irurita, 2003; Doucet et al., 2012), therefore, they can play an important role in creating an environment in which successful breastfeeding can take place (Ryan et al., 2011; Schmied et al., 2011).

For a number of mothers in this study, negative emotions during breastfeeding triggered existing vulnerabilities, such as previous experiences of depression. Thus, employing a person-centred approach to care whereby mothers’ previous experiences, beliefs, expectations and goals are explored allows for a tailored form of support (Hauck and Irurita, 
2003; Palmér et al., 2010; Schmied, et al., 2011). Indeed, the current findings evidence how the strains associated with breastfeeding led mothers to draw on a range of sources and coping strategies to alleviate their distress.

Mothers expressed surprise, disappointment and frustration with not having been prepared for negative embodied emotional sensations and experienced that breastfeeding had been promoted solely in positive terms. While existing knowledge recognises that breastfeeding occurs in a context of physiological, psychological and environmental processes (Hauck and Irurita, 2003), current clinical guidelines (WHO and United Nations International Children’s Emergency Fund [UNICEF], 2003; WHO, 2009) and research scarcely acknowledge psychological, environmental (Ryan et al., 2011) and cultural aspects of breastfeeding (Wiessinger, 1996; Dykes and Flacking, 2010). Perinatal care could more specifically convey the multifaceted nature of breastfeeding and thus avoid setting unrealistic expectations for mothers (Hauck and Irurita, 2003; Schmied et al., 2011; Leeming et al., 2013). There appears to be a discrepancy between existing clinical guidelines (e.g., NICE, 2008) that acknowledge the need for a multifaceted approach to breastfeeding support and mothers' actual experiences of such support.

In addition to formal support, current findings also identify a number of functions that informal peer support groups may have for mothers, such as alleviating guilt and a sense of defectiveness, allowing them to normalise and make sense of their experiences in a safe, shared space. Current clinical guidelines (NICE, 2008; National Health and Medical Research Council [NHMRC], 2012; WHO, 2009) emphasise the usefulness of peer support groups for mothers who struggle with breastfeeding. Thus, raising professionals' awareness and use of the links with informal peer-support groups or forums specific to negative embodied emotional sensations experienced during breastfeeding may play a powerful role in helping mothers understand and be supported in their breastfeeding endeavours. Unlike 
professionals in overstretched services, peer supporters have the time and commitment to develop trusting relationships with mothers (Schmied et al., 2011).

\section{Limitations}

Due to the exploratory nature of this research, a number of limitations were noted. Firstly, current findings offer preliminary qualitative data about the nature and experiences of embodied emotional sensations and maternal psychological responses to these. In line with the choice of a phenomenological research methodology, the sample is deliberately small and homogenous and thus, broad conclusions about maternal experiences of distress during breastfeeding cannot be made. Secondly, while the interviews were dictated by a broad open ended schedule around the research aims, in line with IPA, longer interviews or longitudinal data may have further enhanced the depth of the findings. Third, some differences were identified among the mothers in the sample including the wide range of negative emotions that were reported (e.g., sadness, anxiety and anger) and different mental health history which may have influenced how they viewed their experiences. Finally, while the use of IPA provided important and unique insights into the embodied emotional sensations of the mothers in this study, the use of other qualitative methods, such as narrative methodology, may have included a wider continuum and context of maternal experiences during or even prior to pregnancy and breastfeeding.

\section{Future Research}

One of the highlighted issues expressed by mothers in this study was professionals' lack of knowledge. A future research avenue could explore healthcare professionals’ understanding of psychological breastfeeding problems and what they perceive that mothers experience as barriers to successful breastfeeding. 
In addition, future research could more specifically examine mothers’ perceived support needs around embodied emotional sensations during breastfeeding, in order to inform staff knowledge and services for mothers in the perinatal period.

Moreover, current findings bring into awareness that existing clinical guidelines do not explicitly acknowledge the psychological nature of breastfeeding. Thus, incorporating the importance of psychological, environmental and cultural aspects of support would enrich existing clinical guidelines and practice around infant feeding and maternal well-being.

Finally, a quantitative exploration of the prevalence of emotional distress during breastfeeding followed by a biomedical investigation of such difficulties could further complement and extend the current research.

\section{Conclusion}

This study explored how mothers experience and make sense of embodied emotional sensations and psychological distress emerging during breastfeeding. Mothers in this study viewed such experiences as an unknown part of self that exacerbated existing vulnerabilities. They expressed great disappointment when emotional experiences interfered with their desired breastfeeding relationship, which was intimately linked in with their maternal identity. Applying a psychological lens to such struggles, which have potential biological origins, enabled mothers to accept, own and manage their experiences. However, it was noted that despite breastfeeding involving a number of relational dynamics that are important for mothers who breastfeed, such aspects are rarely the focus of clinical guidelines and practice. In light of this, clinical recommendations encourage healthcare professionals to approach breastfeeding as a holistic experience, involving physiological processes, indeed, but also psychological and environmental. This includes considering the cultural assumptions and norms relating to breastfeeding in western culture which may influence the solutions that mothers choose when breastfeeding becomes physically or emotionally challenging. Potential 
future research could offer a quantitative appraisal of the prevalence of emotional breastfeeding distress, biomedically explore such experiences and qualitatively explore healthcare professionals' understanding of such difficulties. Continued clinical and research activity in this area may help mothers to manage their difficulties during the breastfeeding period, while feeling empowered and supported to make choices that they fundamentally believe are right for them and their children.

\section{References}

Avery, A., Zimmerman, K., Underwood, P. W., Magnus, J. H., 2009. Confident commitment is a key factor for sustained breastfeeding. BIRTH, 26, 141-148.

Borra, C., Iacovou, M., Sevilla, A., 2014. New evidence on breastfeeding and postpartum depression: The importance of understanding women’s intentions. Maternal and Child Health Journal, 18, 897-907.

British Psychological Society, 2010. Code of human research ethics. Leicester: The British Psychological Society.

http://www.bps.org.uk/sites/default/files/documents/code_of_human_research_ethics. pdf

Carter, S. C., 2003. Developmental consequences of oxytocin. Physiology \& Behaviour, 79, 383-397.

Cattaneo, A., 2008. The benefits of breastfeeding or the harm of formula feeding? Journal of paediatrics and child health, 44, 1-2.

Coles, J., 2009. Qualitative study of breastfeeding after childhood sexual assault. Journal of Human Lactation, 25, 317-324.

Cox, S., 2010. Case of Dysphoric Milk Ejection Reflex. Breastfeeding Review, 18, 16-18. 
Creswell, J. W., 2012. Qualitative enquiry and research design: choosing among five approaches. SAGE Publications, California.

Dahlberg, K., 2006. The essence of essences - the search for meaning structures in phenomenological analysis of lifeworld phenomena. International Journal of Qualitative Studies on Health and Well-being 1, 11-19.

Department of Health, 2007. National Service Framework for Children Young People and Maternity Services Standard 11: Maternity Services. London: Department of Health. http://webarchive.nationalarchives.gov.uk/20130107105354/http://www.dh.gov.uk/en /Publicationsandstatistics/Publications/PublicationsPolicyAndGuidance/Browsable/D $\underline{\text { H } 4094336}$

Doucet, S., Letourneau, N., Robertson Blackmore, E., 2012. Support needs of mothers who experience postpartum psychosis and their partners. Journal of Obstetric, Gynecologic, \& Neonatal Nursing, 41, 236-245.

Dykes, F., Flacking, R., 2010. Encouraging breastfeeding: A relational perspective. Early Human Development, 86, 733-736.

Ellet, J., 2011. Narrative and phenomenology as methodology for understanding persistence in art teachers: A reflective journey. Marilyn Zurmuehlin Working Papers in Art Education. http://ir.uiowa.edu/cgi/viewcontent.cgi?article=1407\&context=mzwp

Flower, H, 2003. Adventures in Tandem Nursing: Breastfeeding during Pregnancy and Beyond. La Leche League International, Illinois.

Hanna, P., 2012. Using internet technologies (such as Skype) as a research medium: a research note. Qualitative Research 12, 239-242.

Harper, D., 2012. Choosing a qualitative research method. In Harper, D., Thompson, A. R. (Eds.), Qualitative research methods in mental health and psychotherapy: A guide for students and practitioners. John Wiley \& Sons Ltd, Chichester, UK. pp. 83-97. 
Hauck, Y. L., Irurita, V. F., 2003. Incompatible expectations: The dilemma of breastfeeding mothers. Health Care for Women International, 24, 62-78.

Hegney, D., Fallon, T., O’Brien, M. L., 2008. Against all odds: A retrospective casecontrolled study of women who experienced extraordinary breastfeeding problems. Journal of Clinical Nursing 17, 1182-1192.

Heise, A. M., 2011. Helping professionals understand Dysphoric Milk Ejection Reflex (DMER). http://www.d-mer.org/uploads/English_Professionals_handout_2-11.pdf

Heise, A. M. \& Wiessinger, D., 2011. Dysphoric milk ejection reflex: A case report. International Breastfeeding Journal, 6, 1-6.

Huang, K., Atlas, R., Parvez, F., 2012. The significance of breastfeeding to incarcerated women: An exploratory study. Birth: Issues in Perinatal Care, 39, 145-155.

Kelleher, C. M, 2006. The physical challenges of early breastfeeding. Social Science \& Medicine, 63, 2727-2738.

Klaus, P., 2010. The impact of childhood sexual abuse on childbearing and breastfeeding: The role of maternity caregivers. Breastfeeding Medicine, 5, 141-145.

Kleiman, K. \& Wenzel, A., 2011. Dropping the baby and other scary thoughts: breaking the cycle of unwanted thoughts in motherhood. Routledge, New York \& Hove.

Larsen, J. S., Hall, E. O. C., Agaard, H., 2008. Shattered expectations: When mothers’ confidence in breastfeeding is undermined - a metasynthesis. Scandinavian Journal of Caring, 22, 653-661.

Larsen, J. S., Kronborg, H., 2012. When breastfeeding is unsuccessful - mothers' experiences after giving up breastfeeding. Scandinavian Journal of Caring Sciences, 27, 848-856.

Leeming, D., Williamson, I., Lyttle, S., Johnson, S., 2013. Socially sensitive lactation: Exploring the social context of breastfeeding, 28, 450-468. 
Lewis, S. E., Nicolson, P., 1998. Recognising loss and reconstructing depression. Journal of Reproductive and Infant Psychology, 16, 177-197.

Marshall, J. L., 2011. Motherhood, breastfeeding and identity. Practising Midwife, 14, 16-18.

Marshall, J. L., Godfrey, M., Renfrew, M. J., 2007. Being a 'good mother’: Managing breastfeeding and merging identities. Social Science \& Medicine, 65, 2147-2159.

Murphy, E., 2000. Risk, responsibility, and rhetoric in infant feeding. Journal of Contemporary Ethnography, 29, 291-325.

National Health and Medical Research Council, 2012. Infant Feeding Guidelines: Information for Health Workers. https://www.nhmrc.gov.au/_files_nhmrc/publications/attachments/n56_infant_feeding _guidelines.pdf

National Institute for Health and Clinical Excellence, 2008. Maternal and Child Nutrition. NICE public health guideline 11. http://www.nice.org.uk/guidance/PH11 National Institute for Health and Clinical Excellence, 2014. Antenatal and Postnatal Mental Health: Clinical Management and Service Guidance. NICE clinical guideline 45. http://www.nice.org.uk/guidance/cg192/resources/guidance-antenatal-and-postnatal$\underline{\text { mental-health-clinical-management-and-service-guidance-pdf }}$

Palmér, L., Carlsson, G., Mollberg, M., Nyström, M., 2010. Breastfeeding: An existential challenge - Women's lived experiences of initiating breastfeeding within the context of early home discharge in Sweden. International Journal of Qualitative Studies on Health and Well-being, 5, 5397-5409.

Pancer, S. M., Pratt, M., Hunsberger, B., Gallant, M., 2000. Thinking ahead: Complexity of expectations and the transition to parenthood. Journal of Personality, 68, 253-280.

Phillips, K. F., 2011. First-time breastfeeding mothers: Perceptions and lived experiences with breastfeeding. International journal of childbirth education, 26, 17-20. 
Pietkiewicz, I., Smith, J. A., 2012. A practical guide to using Interpretative Phenomenological Analysis in qualitative research psychology. Czasopismo Psychologiczne, 18, 361-369.

Probst, B., 2015. The eye regards itself: Benefits and challenges of reflexivity in qualitative social work research. Social Work Research, 39, 37-48.

Raphael-Leff, J., 2001. Pregnancy: The Inside Story. Karnac Books, London.

Redshaw, M. \& Henderson, J., 2012. Learning the hard way: Expectations and experiences of infant feeding support. Birth: Issues in Perinatal Care, 39, 21-29.

Ryan, K., Todres, L., Alexander, J., 2011. Calling, permission, and fulfilment: The interembodied experience of breastfeeding. Qualitative Health Research 21, 731742.

Schmied, V., Beake, S., Sheehan, A., McCourt, C., Dykes, F., 2011. Women’s perceptions and experiences of breastfeeding support: A metasynthesis. Birth: Issues in Perinatal Care, 38, 49-60.

Shaw, R. L., 2010. Interpretative phenomenological analysis. In Forrester, M., (Ed.) Doing qualitative research in psychology. SAGE, London.

Smith, J., Dunstone, M., Elliot-Rudder, M., 2009. Health professional knowledge of breastfeeding: Are the health risks of infant formula feeding accurately conveyed by the titles and abstracts of journal articles? Journal of Human Lactation, 25, 350-358.

Smith, J. A., Flowers, P., Larkin, M., 2009. Interpretative phenomenological analysis: theory, method and research. SAGE Publications Ltd, London.

Smith, J. A. \& Osborn, M., 2008. Interpretative Phenomenological Analysis. In Smith, J. A. (Ed), Qualitative psychology: A practical guide to research methods. SAGE Publications Ltd, London. 
Snelgrove, S. R., 2011. Conducting qualitative longitudinal research using interpretative phenomenological analysis. Nurse Researcher, 22, 20-25. http://journals.rcni.com/doi/pdfplus/10.7748/nr.22.1.20.e1277

Sofaer, S., 1999. Qualitative methods: What are they and why use them? Health Services Research, 34, 1101-1118. http://www.hsr.org/

Starks, H. \& Trinidad, S., 2014. Choose your method: A comparison of phenomenology, discourse analysis, and grounded theory. Qualitative Health Research, 17, 1372-1380.

Strathern, L., Fonagy, P., Amico, J., Montague, R. P., 2009. Adult attachment predicts maternal brain and oxytocin response to infant cues. Neuropsychopharamacology, 34, 2655-2666.

Stuebe, A. M., Grewen, L., Meltzer-Brody, S., 2013. Association between maternal mood and oxytocin response to breastfeeding. Journal of Women’s Health 22, 352-361.

Svensson, A., Samuelsson, I., Hellström, A-L, Nolbris, M., 2014. Experiences of Skype communication in education and research: Data collection concerning young children with long-term illness. Early Child Development and Care, 184, 1017-1030.

Thompson, A. R., Harper, D., 2012. Introduction. In Harper, D., Thompson, A. R. (Eds.), Qualitative research methods in mental health and psychotherapy: A guide for students and practitioners. John Wiley \& Sons, Ltd, Chichester, UK. pp. 3-8.

Ussher, J. M., 2003. The role of the premenstrual dysphoric disorder in the subjectification of women. Journal of Medical Humanities 24, 131-146.

Ussher, J. M. \& Perz, J., 2013. PMS as a process of negotiation: Women’s experience and management of premenstrual distress. Psychology \& Health, 28, 909-927.

Uvnäs-Moberg, K., 1998. Oxytocin may mediate the benefits of positive social interaction and emotions. Psychoneuroendocrinology, 23, 819-835. 
Uvnäs-Moberg, K., 2009. Närhetens hormon: Oxytocinets roll i relationer [The hormone of closeness: The role of oxytocin in relationships]. Nature \& Kultur, Stockholm, Sweden.

Wiessinger, D., 1996. Watch your language! Journal of Human Lactation, 12, 1-4.

Wiessinger, D., West, D., Pitman, T., 2010. The Womanly Art of Breastfeeding. Ballantine Books, New York.

World Health Organization, 2009. Infant and young child feeding: Model chapter for textbooks for medical student and allied health professionals. http://whqlibdoc.who.int/publications/2009/9789241597494_eng.pdf

World Health Organization and United Nations International Children’s Emergency Fund, 2003. Global strategy for infant and young child feeding. World Health Organisation, Geneva. http://www.who.int/nutrition/topics/global_strategy/en/ 